\title{
Miele: „Made in Germany aus Österreich” Werk in Bürmoos wird 2. Kompetenzzentrum für Medizintechnik
}

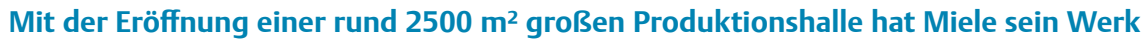
im österreichischen Bürmoos Ende vergangenen Jahres zum zweiten Professional Kompetenzzentrum für Medizintechnik erklärt. 2,8 Millionen Euro investierte das Familienunternehmen aus Gütersloh in die Fertigungshalle für Dampf-Sterilisatoren und Großkammer-Reinigungs- und Desinfektionsanlagen, die zuvor unter der Marke Dirschl vertrieben wurden. Ein zwingender Schritt für die Sparte Medizintechnik von Miele, um für den Bereich Krankenhaushygiene als Systemanbieter die Einrichtung einer gesamten Zentralsterilisation anbieten zu können.

Bereits seit einem halben Jahrhundert lässt der ostwestfälische Haushaltsgerätehersteller - nur einen Katzensprung von der deutschen Grenze im Westen - in Österreich „Miele - Made in Germany“ produzieren. Aus wirtschaftsstrategischen Gründen und unternehmerischer Vorsicht hatten die Enkel der Unternehmensgründer Rudolf Miele und Dr. Peter Zinkann vor 50 Jahren mit dem Werk im Salzburger Land einen Fuß in die damalige EFTA-Zone gesetzt. Damals drohte Europa in 2 Handelsblöcke zu zerfallen. Miele setzte auf Österreich, denn das war Mitglied der EFTA (Europäische Freihandelsassoziation), die 1960 als Gegengewicht zur Europäischen Wirtschaftsgemeinschaft gegründet wurde und eröffnete 1962 im kleinen Städtchen Bürmoos im Flachgau ein Werk.

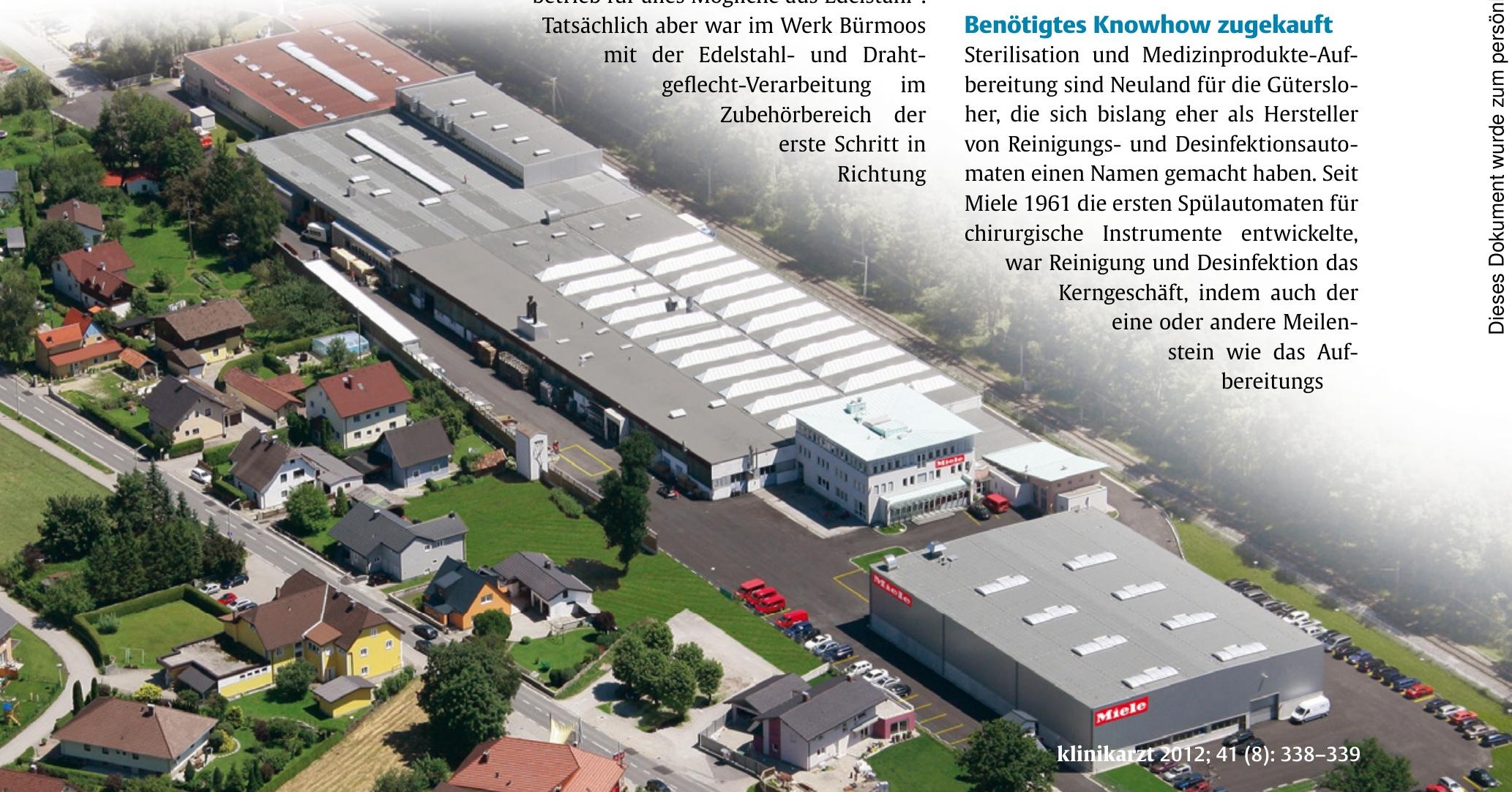

\section{Spezialist für Reinigungs- und}

\section{Desinfektionsautomaten}

Dieser erste und lange Zeit einzige MieleProduktionsstandort außerhalb von Gütersloh im Norden der Festspielstadt Salzburg sollte zweites europäisches Standbein sein. Auf $4600 \mathrm{~m}^{2}$ wurden zunächst Wäschezentrifugen, später dann Waschautomaten gebaut. Als Spezialist im Edelstahlbereich produzierte Miele Bürmoos dann Blenden und Verkleidungsteile für Hausgeräte, 1967 kam die Produktion von Einsätzen und Körben aus rostfreiem Edelstahl für Desinfektions- und Reinigungsautomaten der MedizintechnikSparte von Miele Professional dazu.

„Miele-Werk in Bürmoos ist ein Sanierungsfall", unkte im Februar 2008 das in Wien erscheinende WirtschaftsBlatt über den angeblich „defizitären Zulieferbetrieb für alles Mögliche aus Edelstahl“. mit der Edelstahl- und Drahtht-Verarbeitung im rbereich der Richtung

Medizintechnik gemacht worden und in Gütersloh reifte der Plan, das Werk in Österreich zu einem zweiten Kompetenzzentrum für die Miele Professional Medizintechnik zu machen.

Das Geschäft mit den Reinigungs- und Desinfektionsautomaten hatte sich als Wachstumsmarkt entpuppt; in den vergangenen Jahren war der Umsatz von Miele Professional überproportional gestiegen. Miele hatte bereits für rund 17 Millionen Euro ein Entwicklungs-, Fertigungs- und Testzentrum in Bielefeld eingerichtet. Etwa 10 Millionen Euro wurden zudem in Österreich investiert, um neben dem Werk in Bielefeld ein zweites Kompetenzzentrum für Medizintechnik zu errichten. Während in Bielefeld ein- und zweitürige Großraumdesinfektoren sowie die Reinigungs- und Desinfektionsautomaten für das Seriengeschäft hergestellt werden, produziert man in Bürmoos Dampf-Sterilisatoren und die Großkammer-Reinigungs- und Desinfektionsanlagen. Für Dr. Markus Miele war es einmal die hohe Kompetenz, die das Werk in Bürmoos in der Edelstahlverarbeitung hatte und zum anderen das Platzangebot des 40000 Quadratmeter großen Werksgelände, das für Bürmoos sprach.

\section{Benötigtes Knowhow zugekauft}

Sterilisation und Medizinprodukte-Aufbereitung sind Neuland für die Gütersloher, die sich bislang eher als Herstelle Reinigungs- und Desinfektionsauto1961 die ersten Spülautomaten fü war Reinigung und Desinfektion das eschäft, indem der andere $x<<\ldots$ 
programm Oxivario Plus für Instrumente, deren Reinigung laut Robert-Koch-Institut als kritisch eingestuft werden muss gesetzt werden konnte. Sterilisatoren wurden, wenn nötig, zugekauft. Zum echten Systemanbieter, der eine gesamte Zentralsterilisation anbieten, bauen und warten kann, fehlte der Medizintechniksparte bei Miele noch die Segmente der Sterilisation und Medizinprodukte-Aufbereitung.

Um an das entsprechende Knowhow und die wichtigen Patente heranzukommen, kaufte Miele zunächst Teile des niederländischen Steriherstellers Davenport, der dann in Miele aufging. Der Durchbruch zum Systemanbieter, der alles komplett aus einer Hand liefert, kam 2009, als Miele den Bereich Containerwaschanlagen der Marke Dirschl im bayerischen Erding übernahm. Das kleine mittelständische Unternehmen mit rund 20 Mitarbeitern, das seit 2007 zur BHT-Unternehmensgruppe gehörte, hatte genau das, was Miele noch fehlte: Sachkompetenz im Bau von Container- und Transportwagen-Waschanlagen. Dirschl hatte sich auf die Produktion von Großkammerwaschanlagen für
Krankenhäuser spezialisiert, in denen vom Nachtschränkchen, über Sterilgutcontainer bis hin zu größeren Transportwagen und Betten alles gewaschen werden kann. Bereits in der Vergangenheit hatten Miele und Dirschl bei verschiedenen Projekten zusammengearbeitet, es kam also nicht überraschend, dass die BHT Hygienetechnik $\mathrm{GmbH}$, die sich von dem Geschäftsbereich „Dirschl“ trennen wollte, an Miele verkaufte.

\section{Werk im letzten Herbst eröffnet}

Erding war für einen Ausbau der Produktion zu klein. Nachdem zunächst die Herstellung der Containerwaschanlagen dort weiter lief, produziert Miele seit dem Januar 2010 im Werk Bürmoos im eigenen Namen „Container- \& TransportwagenWaschanlagen“. Einige Dirschl-Mitarbeiter konnte man für den neuen Betriebsstandort in Bürmoos gewinnen, mit deren Kenntnissen jetzt die Entwicklung und Produktion im Anlagenbau ausgebaut wird. Heute werden in Bürmoos Großund Klein-Sterilisatoren sowie Containerund Transportwagen-Waschanlagen entwickelt. Die Werksfläche wurde verdop-

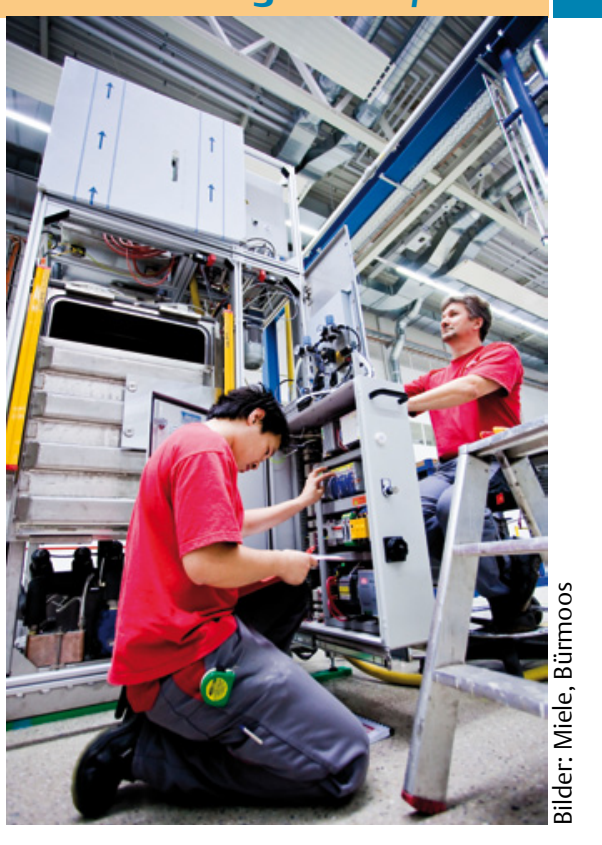

pelt, die Mitarbeiterzahl soll von 246 auf 300 steigen und beim Umsatz peilt man in Bürmoos 50 statt zuletzt 25,5 Millionen Euro an - da ließ es sich die landespolitische Prominenz vergangenen Herbst nicht nehmen, das so geadelte Werk zusammen mit der aus Gütersloh angereisten Unternehmensführung zu eröffnen.

Anne Marie Feldkamp, Bochum 